\title{
Die Experimentelle Philosophie und unsere Verwendung sprachlicher Ausdrücke
}

\section{1 \\ Einleitung}

Das Stichwort »Experimentelle Philosophie« wird für gewöhnlich in erster Linie mit Philosophinnen und Philosophen assoziiert, die Umfragen durchführen, um herauszufinden, welches die etablierte Verwendung philosophisch interessanter Begriffe wie Wissen oder Absichtlichkeit ist, oder mit Moralphilosophinnen und -philosophen, die Laien beispielsweise dazu befragen, wie man sich in einem bestimmten »trolley case« verhalten soll, um herauszufinden, welches unsere alltäglichen Moraltheorien sind. Tatsächlich ist die Experimentelle Philosophie jedoch ein weites Feld, innerhalb dessen mindestens vier verschiedene Strömungen zu unterscheiden sind. Drei davon werden in einem vielzitierten Artikel von Thomas Nadelhoffer und Eddy Nahmias unter den Labels »Experimentelle Analyse«, »Experimenteller Deskriptivismus « und »Experimenteller Restriktionismus« besprochen.

Vertreterinnen und Vertreter der Experimentellen Analyse verfolgen das eingangs erwähnte Projekt: Sie erheben die Intuitionen einer statistisch signifikanten Gruppe von typischerweise nicht philosophisch ausgebildeten Personen, mit dem Ziel, zu erkennen, welche philosophischen Positionen z. B. in Bezug darauf, was Wissen ist oder unter welchen Bedingungen eine Handlung absichtlich ausgeführt wird - mit dem Commonsense übereinstimmen. Dabei gehen sie davon aus, so Nadelhoffer und Nahmias, dass die Übereinstimmung mit dem Commonsense wenigstens ein Prima-facieArgument für die Korrektheit einer Theorie ist. ${ }^{1}$

Vertreterinnen und Vertreter des Experimentellen Deskriptivismus interessieren sich demgegenüber zunächst einmal für Intuitionen um ihrer selbst willen. Sie untersuchen, welche Intuitionen die Leute haben, welche kognitiven Mechanismen diesen Intuitionen zugrunde liegen und wodurch sie sich beeinflussen lassen. In einem zweiten Schritt versuchen sie, basierend auf den Ergebnissen zu den Ursachen und Beeinflussungsfaktoren von Intuitionen, ein Urteil in Bezug darauf zu fällen, welchen Intuitionen wir trauen können und welchen nicht; das heißt: welchen Anforderungen Intuitionen genügen

1 Vgl. Nadelhoffer/Nahmias 2007, S. $126 f$.

(C) MENTIS VERLAG, 2020 | DOI:10.30965/9783957437259_009

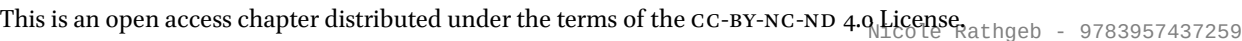


müssen, um zur Stützung philosophischer Positionen angeführt werden zu können. ${ }^{2}$

Vertreterinnen und Vertreter des Experimentellen Restriktionismus bezweifeln, dass Intuitionen überhaupt einen epistemischen Wert haben, und berufen sich dabei unter anderem auf Studien, die zeigen sollen, dass sich die Intuitionen von Personen mit unterschiedlichem kulturellem oder sozioökonomischem Hintergrund wie auch von Personen verschiedenen Geschlechts oder Alters stark voneinander unterscheiden. ${ }^{3}$ Außerdem sollen diese Studien belegen, dass die Reaktionen von Personen auf Gedankenexperimente beispielsweise mit der Reihenfolge der vorgetragenen Beispiele variieren oder mit anderen Parametern, die für die gestellten Fragen (wenigstens auf den ersten Blick) keine Relevanz haben. ${ }^{4}$ So sind Probandinnen und Probanden zum Beispiel ceteris paribus desto mehr dazu geneigt, über einen Akteur zu sagen, dass er für seine Handlungen moralisch verantwortlich ist, je schlimmer seine Handlungen sind. ${ }^{5}$

Eine vierte philosophische Strömung, die mitunter als Spielart der Experimentellen Philosophie gehandelt wird, aber in der Klassifikation von Nadelhoffer und Nahmias keine Berücksichtigung findet, ist die Strömung, deren Vertreterinnen und Vertreter selbst echte Experimente (und nicht bloß Befragungen $^{6}$ ) durchführen und beispielsweise mit Eyetrackern oder bildgebenden Verfahren der Neurowissenschaften arbeiten. ${ }^{7}$ Ich werde diese Strömung nachfolgend auch als »Empirische Philosophie« bezeichnen.

Im vorliegenden Kapitel werde ich mich vor allem mit der Experimentellen Analyse und dem Experimentellen Restriktionismus befassen, da von den Vertreterinnen und Vertretern dieser beiden Auffassungen am deutlichsten Position gegen die »im Lehnstuhl« betriebene Ordinary Language-Philosophie

2 Vgl. Nadelhoffer/Nahmias 2007, S. 127f. Im Sinne dieses Unterfangens äußern sich beispielsweise Nichols/Knobe 2007, S. 663f., 678 und Fischer 2014, S. 324.

3 Weinberg, Nichols und Stich sind sogar der Auffassung, dass bereits die (logische, nomologische und/oder psychologische) Möglichkeit divergierender Intuitionen ein Problem darstellt für die Verwendung von Intuitionen zur Stützung philosophischer Thesen. Dass auch noch gezeigt werden könne, dass solche Divergenzen tatsächlich bestünden, sei für ihre Argumentation begrüßenswert, aber letztlich nicht notwendig. Vgl. Weinberg/Nichols/ Stich 2008 [2001], S. 22f.

4 Vgl. Nadelhoffer/Nahmias 2007, S. 128, Grundmann/Horvath/Kipper 2014, S. 12f., Alexander/ Weinberg 2007, S. 61-66, Weinberg/Nichols/Stich 2008 [2001], S. $23-34$.

5 Vgl. Nichols/Knobe 2007, S. 675 f.

6 Man beachte allerdings, dass in der Experimentellen Philosophie teilweise mit einem Experiment-Begriff operiert wird, der besonders kontrollierte Befragungen umfasst, aber nicht das, was üblicherweise als »Umfrage« bezeichnet wird. Vgl. z. B. Fischer 2014, S. 323, Fußnote 5.

7 Vgl. z. B. Metzinger/Windt 2014, Prinz 2008. 
bezogen wird, die ich verteidige. Sowohl vonseiten der Experimentellen Analyse als auch vonseiten des Experimentellen Restriktionismus wird die OLP so dargestellt, dass sie ihre Erwägungen auf Annahmen darüber abstützt, welche Intuitionen »wir« haben - wobei in der Regel nicht weiter spezifiziert werde, welche Gruppe mit »wir « gemeint sei. ${ }^{8}$ Dass die Vertreterinnen und Vertreter der OLP sich mit »wir « ausschließlich auf ihre eigenen Intuitionen beziehen wollen (bzw. auf diejenigen von ihnen selbst und den Philosophinnen und Philosophen, mit denen sie zusammenarbeiten), sei unplausibel, da die Berufung auf persönliche Intuitionen offensichtlich nicht dazu geeignet sei, jemanden von der Wahrheit einer philosophischen These zu überzeugen. ${ }^{9}$ Viel plausibler sei, dass die Vertreterinnen und Vertreter der OLP mit »wir« den Leser und die Leserin mit-meinen und davon ausgehen, dass sie ihre Intuitionen mit einer größeren Gruppe, zum Beispiel mit einer Mehrheit ihrer Sprachgemeinschaft oder sogar der Menschheit teilen. ${ }^{10}$ An diesem Punkt setzt die Kritik vonseiten der Experimentellen Philosophie an:

Vertreterinnen und Vertreter der Experimentellen Analyse machen geltend, dass wir nicht ohne empirische Untersuchung wissen können, in welchem Maß unsere Intuitionen von anderen Personen geteilt werden, und dass wir eine angemessene Stichprobe von Personen befragen müssen, um begründete Urteile in Bezug darauf treffen zu können, welches die etablierten Intuitionen sind. Im Sinne dieser Argumentation wird die Experimentelle Analyse als Verbesserung der OLP dargestellt.. ${ }^{11}$

Die Kritik an der OLP vonseiten des Experimentellen Restriktionismus geht noch einen Schritt weiter: Nicht nur können wir nicht aus dem Lehnstuhl beurteilen, welches die Intuitionen sind, die eine Mehrheit der Bevölkerung besitzt, sondern Studien zeigen sogar, dass es zwischen den Intuitionen verschiedener Personen und Gruppen gravierende Unterschiede gibt. So werden Gettier-Fälle zum Beispiel von einer Mehrheit ostasiatischer Probandinnen und Probanden als Fälle von Wissen beurteilt, während Westlerinnen und Westler überwiegend der Auffassung sind, dass die Akteure in Gettier-Fällen nicht über Wissen verfügen. ${ }^{12}$ Da nichts dafür spricht, dass die westlichen den

8 Vgl. Alexander/Weinberg 2007, S. 57, Weinberg/Nichols/Stich 2008 [2001], S. 18.

9 Vgl. Alexander/Weinberg 2007, S. 57 f.

10 Vgl. Alexander/Weinberg 2007, S. 57, 6o. Als alternative Auffassung besprechen Alexander und Weinberg die Idee, mit »wir« seien alle professionellen Philosophinnen und Philosophen gemeint. Ein entsprechender »Elitismus« lässt sich aber, so Alexander und Weinberg, nicht stichhaltig begründen. Vgl. Alexander/Weinberg 2007, S. 58-60.

11 Vgl. Alexander/Weinberg 2007, S. 61, Prinz 2008, S. 193f., Metzinger/Windt 2014, S. 305.

12 Vgl. Weinberg/Nichols/Stich 2008 [2001], S. $29 f$. 
ostasiatischen Intuitionen überlegen sind (oder umgekehrt), ${ }^{13}$ und es nicht vernünftig wäre, anzunehmen, dass immer das richtig ist, was die Mehrheit der gesamten Menschheit sagt, ${ }^{14}$ sollten wir insgesamt die Idee aufgeben, dass uns Intuitionen Aufschluss über philosophische Wahrheiten geben können. ${ }^{15}$

Die Kritik an der OLP vonseiten der Experimentellen Philosophie läuft also auf die Behauptungen hinaus,

(E1) dass wir von unseren Lehnstuhl-Intuitionen nicht auf die Intuitionen der restlichen Bevölkerung schließen können resp.

(E2) dass Intuitionen prinzipiell nicht als philosophische Evidenz geeignet sind.

Was Intuitionen sind, wird in den Texten von Vertreterinnen und Vertretern der Experimentellen Philosophie unterschiedlich bestimmt, meistens aber ungefähr als starke Neigung dazu, ein bestimmtes Urteil für wahr zu halten, die nicht auf einer (bewussten) Herleitung dieses Urteils aus anderen Urteilen beruht. ${ }^{16}$ So definiert, sind Intuitionen aber nichts, worauf in Argumenten einer adäquat aufgefassten OLP Bezug genommen wird. Vertreterinnen und Vertreter der OLP berufen sich nicht auf Intuitionen, sondern auf ihr Sprachgefühl bzw. ihre Sprachkompetenz. In Beurteilungen von Gedankenexperimenten der OLP finden wir für gewöhnlich keine Aussagen der Art »Wir haben die Intuition, dass der betreffende Akteur über Wissen verfügt«, sondern eher Aussagen wie »Wir würden sagen, dass der Akteur über Wissen verfügt«. Was wir sagen oder nicht sagen würden, gibt uns darüber Aufschluss, welches die Bedingungen für das Vorliegen von Wissen sind bzw. darüber, was Wissen ist.

Auf eine auf diese Weise verstandene OLP sind jedoch die obigen Argumente der Experimentellen Philosophie problemlos übertragbar:

$\left(\mathrm{E}^{*}\right) \quad$ Wir können daraus, wie wir selbst ein Wort verwenden, nicht darauf schließen, wie andere Sprecherinnen und Sprecher es verwenden, und müssen, um zu Aussagen der Art »Wir würden sagen, dass das ein Fall von Wissen ist « berechtigt zu sein, empirische Studien durchführen.

$\left(\mathrm{E2}^{*}\right) \quad$ Wie sich herausgestellt hat, verwenden verschiedene Personen beispielsweise das Wort »wissen « (oder seine Entsprechungen in anderen Sprachen) unterschiedlich, und es gibt keinen Grund zur Annahme, dass unsere Weise, das Wort zu verwenden, eine größere Aussagekraft in Bezug darauf hat, was Wissen tatsächlich ist, als die anderen

13 Vgl. Knobe/Nichols 2008, S. 11, Nadelhoffer/Nahmias 2007, S. 128.

14 Vgl. Knobe/Nichols 2008, S. 6.

15 Vgl. Alexander/Weinberg 2007, S. 66f., Weinberg/Nichols/Stich 2008 [2001], S. 21-23, 40, Mallon/Machery/Nichols/Stich 2009, S. 351f.

16 Vgl. z. B. Grundmann/Horvath/Kipper 2014, S. 11, Fußnote 3, Sinnott-Armstrong 2008, S. 209, Brendel 2014, S. 190. 
Verwendungsweisen. Tatsachen in Bezug darauf, was wir sagen würden, sind also nicht als philosophische Evidenz geeignet.

\section{Begriffliche und empirische Fragen}

Vertreterinnen und Vertreter der Experimentellen Philosophie würden allerdings gegen eine OLP, die sich nicht auf Intuitionen abstützt, sondern darauf, was wir sagen würden, nicht nur die Einwände $\left(\mathrm{E}_{1}^{*}\right)$ oder $\left(\mathrm{E}_{2}{ }^{*}\right)$ vorbringen, sondern scheinen in der Regel auch nicht anzunehmen, dass die Befragungen, die sie durchführen, nur das Sprachgefühl der Probandinnen und Probanden prüfen bzw. dass es in den Gedankenexperimenten, die sie verwenden, nur darum geht, wie wir bestimmte sprachliche Ausdrücke gebrauchen. Betrachten wir einige Beispiele für Fragen, mit denen die Teilnehmerinnen und Teilnehmer von Studien der Experimentellen Philosophie konfrontiert wurden:

(i) Ist es moralisch zulässig oder sogar geboten, einen dicken Mann von einer Brücke zu stoßen, um einen Zug zu stoppen, der andernfalls fünf Personen überrollen würde ${ }^{17}$

(ii) Bill lebt in einer Welt, in welcher alles, was passiert, - auch menschliche Handlungen - komplett kausal determiniert ist durch die Dinge, die zuvor passieren. Eines Tages tötet Bill seine Frau und seine Kinder, um mit seiner Sekretärin zusammen sein zu können. Ist er für diese Handlung moralisch verantwortlich? ${ }^{18}$

(iii) Pat zeigt im Zoo auf ein Zebra und sagt: »Das ist ein Zebra.« Er befindet sich allerdings so weit von dem Tier entfernt, dass er ein Zebra nicht von einem als Zebra getarnten Maultier unterscheiden könnte. Weiß Pat, dass das Tier, das er sieht, ein Zebra ist? ${ }^{19}$

(iv) Um ein Würfelspiel zu gewinnen, muss Milt mit zwei Würfeln beim ersten Versuch eine Eins würfeln. Da es ihm wichtig ist zu gewinnen, will Milt unbedingt zwei Einsen würfeln. Seine Chancen stehen 1 zu 36. Tatsächlich würfelt Milt beim ersten Versuch mit beiden Würfeln eine Eins und gewinnt das Spiel. Hat Milt absichtlich zwei Einsen gewürfelt? ${ }^{20}$

17 Das Beispiel stammt aus Jarvis Thomson 1976, S. 207f. Experimentelle Studien zu TrolleyFällen wurden beispielsweise von Zamzow und Nichols (2009) durchgeführt.

18 Vgl. Nichols/Knobe 2007, S. $669 f$.

19 Vgl. Weinberg/Nichols/Stich 2008 [2001], S. 33. Das Beispiel stammt aus Dretske 1970, S. 1015 f.

20 Vgl. Cushman/Mele 2008, S. 186. 
(v) John weiß über Gödel nur, dass er den so genannten »Unvollständigkeitssatz« bewiesen hat. Nehmen wir an, dieser Satz wäre eigentlich nicht von einem Mann namens »Gödel«, sondern von einem Mann namens "Schmidt« bewiesen worden, dessen Manuskript ein Mann namens »Gödel« gestohlen und publiziert hätte. Würde John, wenn er den Namen »Gödel« verwendet, dann über die Person sprechen, die den Unvollständigkeitssatz bewiesen hat, oder über die Person, die das Manuskript gestohlen und unter seinem eigenen Namen publiziert hat?21

Mit dem Fall (i) werde ich mich in der vorliegenden Abhandlung nicht näher befassen. Ich gehe davon aus, dass es sich bei der in (i) gestellten Frage nicht um eine begriffliche Frage handelt und dass also eine Klärung der in (i) verwendeten Begriffe und namentlich eine Klärung des Begriffs der moralischen Zulässigkeit oder des moralischen Gebotenseins nicht dafür hinreichend wäre, sie zu beantworten.

Demgegenüber bin ich der Auffassung, dass es sich bei den Fällen (ii) bis (v) um begriffliche Probleme handelt. Dies ist bei (v) relativ offensichtlich, da darin augenscheinlich zur Debatte steht, wie bestimmte sprachliche Ausdrücke, nämlich Eigennamen funktionieren.

Dafür, dass auch die in (ii) bis (iv) gestellten Fragen durch eine Klärung unserer Begriffe $\mathrm{zu}$ beantworten sind, möchte ich nachfolgend nicht argumentieren; ich möchte lediglich darlegen, wie ich diese Fragen verstehe.

Die Frage in (ii), ebenso wie die allgemeinere Frage, ob Akteure in deterministischen Szenarien für ihre Handlungen moralisch verantwortlich sein können, ist eine Frage nach der begrifflichen Konsistenz zweier Beschreibungen. ${ }^{22}$ Es geht darum, ob die Beschreibung einer Welt $w_{l}$ als deterministisch gleichzeitig wahr sein kann wie die Behauptung, ein Akteur sei für eine Handlung in $w_{1}$ moralisch verantwortlich. Sie ist in dieser Hinsicht vergleichbar mit der Frage, ob der Satz »a ist die Schwester des Großvaters von $b$ « gleichzeitig wahr sein kann wie »b ist die Nichte des Sohnes von a«. Mit dieser Frage konfrontiert, würden wir nicht auf die Idee kommen, eine Umfrage zurate zu ziehen, und die mutmaßliche Tatsache, dass verschiedene Sprecherinnen und Sprecher die Frage unterschiedlich beantworten würden, legt auch nicht nahe, dass es sich dabei um eine Meinungsfrage handelt oder dass es verschiedene Auffassungen der involvierten Verwandtschaftsbeziehungen gibt. Beide Fragen, sowohl diejenige zur Kompatibilität von Determinismus und moralischer Verantwortlichkeit als auch diejenige zu

21 Vgl. Machery/Mallon/Nichols/Stich 2004, S. B6. Das Beispiel stammt aus Kripke 1980, S. 83f.

22 Zumindest ist dies dann der Fall, wenn wir die Behauptung, jemand sei für eine Handlung moralisch verantwortlich, als wahrheitsfähig auffassen. 
den Verwandtschaftsbeziehungen, sind auf der Grundlage begrifflicher Erwägungen zu beantworten - und beide Fragen können aus dem Lehnstuhl beantwortet werden; auf diesen Punkt werde ich weiter unten zurückkommen. ${ }^{23}$

Die Fragen in (iii) und (iv) sind Fragen nach der Verwendung von »wissen « und »absichtlich«. Demgegenüber sind die Fragen

(vi) »Weiß Klaus, dass wir heute zusammen essen gehen?« oder

(vii) »Hat Hannah den Pullover absichtlich in Größe XL gekauft?«, geäußert in gewöhnlichen Situationen, keine Fragen nach der Verwendung dieser Wörter, sondern Fragen danach, was der Fall ist resp. war. Der Unterschied ist, dass wir im Falle von (iii) und (iv) über alle Aspekte der fraglichen Situationen informiert sind, die für die Beantwortung der Fragen relevant sind (oder zumindest wäre es ein Fehler in der Beschreibung eines Gedankenexperiments, wenn sie nicht über alle relevanten Aspekte des Szenarios Aufschluss geben würde ${ }^{24}$ ), und es nur noch darum geht, wie wir eine so geartetes Szenario beschreiben würden, während uns, wenn wir die Fragen (vi) und (vii) stellen, Informationen zu den entsprechenden Situationen fehlen und wir z. B. nicht wissen, ob jemand Klaus angerufen hat, um ihm Bescheid zu geben, oder ob Hannah aus irgendeinem Grund einen übergroßen Pulli benötigt hat.

Manche Vertreterinnen und Vertreter der Experimentellen Philosophie würden mir darin zustimmen, dass sie sich mit begrifflichen Fragen befassen. Häufig werden in der Literatur der Experimentellen Philosophie allerdings begriffliche Fragen oder Aussagen nicht als solche erkannt. Dies passiert am häufigsten bei Vertreterinnen und Vertretern der Empirischen Philosophie. So schreiben zum Beispiel Metzinger und Windt, dass die in Umfragen der Experimentellen Philosophie verwendeten Fragen (und zwar nicht nur die moralphilosophischen) auf die Abfrage von Meinungen gerichtet seien. ${ }^{25}$ Sie selbst setzen sich unter anderem mit der Frage auseinander, ob eine bestimmte, primitive Form des Selbstbewusstseins »denkbar« wäre, und meinen:

23 Bestimmte Philosophinnen und Philosophen würden sagen, in (ii) werde eine Frage nicht zur begrifflichen, sondern zur metaphysischen Kompatibilität von Determinismus und moralischer Verantwortlichkeit gestellt. Ich werde hier nicht dafür argumentieren, dass diese Auffassung falsch ist. So oder so impliziert metaphysische Kompatibilität begriffliche Kompatibilität, deshalb sollten sich auch der Metaphysiker und die Metaphysikerin dafür interessieren, ob die oben genannten Beschreibungen von $w_{l}$ begrifflich konsistent sind oder nicht.

24 Dies gilt zumindest für relevante Aspekte, die nicht offensichtlich sind bzw. als allgemein bekannt vorausgesetzt werden können. Vgl. dazu den Abschnitt 4.3.2 des Kapitels 6 des vorliegenden Buchs.

25 Vgl. Metzinger/Windt 2014, S. 282. Darauf, wieso es in diesem Zusammenhang problematisch sein kann, von »Meinungen«, »Ansichten« und Ähnlichem zu sprechen, komme ich weiter unten zurück. Vgl. Fußnote 36 . 
Ob uns dies intuitiv plausibel erscheint, kann zur Klärung dieser Fragen nichts beitragen, sondern sagt uns lediglich etwas darüber, ob wir solche Erlebnisse mental simulieren können, also ob sie für uns in den Bereich der phänomenalen Möglichkeit fallen. ${ }^{26}$

Vermutlich würden Metzinger/Windt zustimmen, dass die Formulierung mit »denkbar« etwas unglücklich gewählt ist, wenn dafür argumentiert werden soll, dass die Frage nur mittels empirischer Studien beantwortet werden kann. Aber wenn wir die Frage umformulieren und »denkbar« durch »möglich« ersetzen - und mit »Es ist möglich, dass $p$ « so etwas meinen wie: »Wir können $p$ irgendwie verursachen « - dann ist es nach wie vor problematisch, zu behaupten, die Frage, ob diese primitive Form des Selbstbewusstseins möglich sei, sei gleichzeitig sowohl empirisch als auch philosophisch. Die Frage ist bei Metzinger/Windt der folgenden, allgemeineren Frage untergeordnet:

(viii) Was ist die einfachste Form von Selbstbewusstsein? Was ist beim Menschen die kleinste Menge an hinreichenden Bedingungen für die Entstehung des phänomenalen Selbst, also des Gefühls, ein Selbst zu sein? ${ }^{27}$

Diese Frage wird von ihnen wie folgt beantwortet:

Das Gefühl der »Meinigkeit« für einen räumlich situierten Körper als Ganzem - also das Erleben, einen Körper zu besitzen und sich mit diesem zu identifizieren - ist hinreichend für minimales, nichtbegriffliches Selbstbewusstsein, und dieses Gefühl kann selektiv auch in solchen Situationen experimentell manipuliert werden, in denen die Versuchsteilnehmer sich absolut passiv verhalten und sich dieser Manipulation bewusst sind. Demgegenüber ist Agentivität - im Sinne von motorischer Kontrolle, willentlicher Aufmerksamkeitslenkung oder auch kognitiver Selbstbezugnahme - keine notwendige Bedingung für die Entstehung von Selbstbewusstsein. Wenn diese Interpretation stimmt, dann hat diese Studie ${ }^{28}$ einen Erkenntnisfortschritt für philosophische Theorien des Selbstbewusstseins ermöglicht. ${ }^{29}$

26 Metzinger/Windt 2014, S. 309.

27 Vgl. Metzinger/Windt 2014, S. 308.

28 Die Rede ist von einer Studie, in welcher ein 3 D-Bild von Testpersonen zwei Meter vor ihnen stehend eingeblendet wird. Die Testpersonen werden dann am Rücken gestreichelt und sehen gleichzeitig, wie ihr 3 D-Bild am Rücken gestreichelt wird. Dies soll den Effekt haben, dass die Probandinnen und Probanden sich körperlich mit ihrem $3 \mathrm{D}$-Bild identifizieren; verbindet man ihnen zum Beispiel die Augen, dreht und verschiebt sie im Raum und ihnen trägt ihnen auf, wieder zurück zu ihrem Ausgangspunkt zu gehen, so bewegen sie sich näher zu dem Punkt hin, an welchem zuvor ihr 3D-Bild eingeblendet war (vgl. Metzinger/Windt 2014, S. 310f.).

29 Metzinger/Windt 2014, S. 311. 
Das, was an dieser Darlegung philosophisch ist, nämlich die Idee, dass das Erleben, einen Körper zu besitzen und sich mit ihm zu identifizieren, hinreichend für Selbstbewusstsein ist, hängt nicht ab von empirischen Befunden. Der Wahrheitswert dieser Behauptung verändert sich nicht in Abhängigkeit davon, wie ein solches Erleben verursacht werden kann. Das Urteil, dass dieses Erleben auf bestimmte Weise erzeugt werden kann, ist demgegenüber rein empirisch, und hat keinen Einfluss auf die Beantwortung der Frage (viii), sofern diese als philosophische Frage aufgefasst wird (nämlich ungefähr im Sinne von: Welche Eigenschaften muss ein Wesen mindestens besitzen, damit wir ihm korrekterweise ein Selbstbewusstsein zuschreiben können?).

Der Behauptung von Metzinger und Windt, dass die oben genannte empirische Studie die philosophischen Theorien des Selbstbewusstseins weitergebracht hat, kann höchstens in dem Maße zugestimmt werden, in welchem die Studie Anlass gegeben hat zu einer bestimmten philosophischen Frage - nämlich: »Zählt das Erleben, einen Körper zu besitzen und sich mit ihm zu identifizieren, bereits als eine Form von Selbstbewusstsein? ${ }^{30}{ }^{30}$ Diese Frage selbst ist aber nicht empirisch. Umgekehrt macht der Umstand, dass eine bestimmte philosophische Überlegung Anlass zu einer empirischen Studie gibt, diese Studie dann auch nicht (teilweise) philosophisch. ${ }^{31}$ Metzinger und Windt äußern sich zu diesem Punkt wie folgt:

Handelt es sich bei dieser Studie aber nicht einfach um eine gewöhnliche empirische Studie, der im Nachhinein eine philosophische Interpretation übergestülpt wurde? Nein, denn die Fragestellung, das epistemische Ziel, kam aus der Philosophie. Die Ausgangsfrage lautete: Ist es möglich, eine globalisierte »Ganzkörper-Variante« der Gummihand-Illusion (RHI; rubber hand illusion) zu erzeugen? Und falls ja, welche Konsequenzen hätte dies für Theorien des Selbstbewusstseins und für eine präzisere begriffliche Isolierung seiner Minimalbedingungen?32

Hier ist die empirische von der philosophischen Frage sogar explizit getrennt. Ob sich die Ganzkörper-RHI (z. B. auf die in Fußnote 28 beschriebene Weise) erzeugen lässt, ist eine rein empirische Frage. Welche Konsequenzen dies für die Philosophie des Geistes hätte, lässt sich, wie bereits an der Formulierung der Frage im Konjunktiv erkennbar ist, ganz unabhängig davon beantworten, ob die empirische Frage bejaht oder verneint wird: Die Frage ist nämlich: Wie

30 Vgl. zur fruchtbaren Zusammenarbeit von Philosophinnen und empirischen Wissenschaftlern auch das, was Hans-Johann Glock in Glock 2013 und Glock 2017a unter dem Stichwort »Impure Conceptual Analysis« beschreibt.

31 Das Gegenteil behaupten Metzinger/Windt (2014, S. 312).

32 Metzinger/Windt 2014, S. 311f. 
würden wir einen solchen Fall beschreiben? Würde er als ein Fall von Selbstbewusstsein zählen oder nicht? Und um diese Frage zu beantworten, müssen wir keine empirische Studie durchführen.

Aber der Fehler der Vermischung begrifflicher mit nicht-begrifflichen Fragen und Aussagen unterläuft auch Vertreterinnen und Vertretern des Experimentellen Restriktionismus und der Experimentellen Analyse. Weinberg, Nichols und Stich äußern sich in ihrem berühmten Artikel »Normativity and Epistemic Intuitions« zum Beispiel ausdrücklich skeptisch in Bezug auf die Idee, dass verschiedene Teilnehmerinnen und Teilnehmer an ihren Studien unterschiedliche Wissensbegriffe besitzen, ${ }^{33}$ schlagen aber andererseits zur Erklärung der Diversität der erhobenen »Wissensintuitionen« vor, es gebe zwischen den Probandinnen und Probanden (unter anderem) Unterschiede darin,

a) in welchem Ausmaß es ihnen zufolge für die Wahrheit von $» S$ weiß, dass $p$ « relevant ist, ob es Alternativen zu $p$ gibt, die $S$ nicht ausschließen kann, und

b) von welchen Mindeststandards für das Vorliegen von Wissen sie ausgehen. ${ }^{34}$

Sie verkennen dabei, dass sowohl (a) als auch (b) Faktoren sind, die für den Wissensbegriff konstitutiv sind. Zwei Personen können nicht einen Wissensbegriff teilen, aber unterschiedliche Mindeststandards für das Vorliegen von Wissen haben. ${ }^{35}$ Denn unter welchen Bedingungen jemand Wissen zuzuschreiben bereit ist, ist mitentscheidend dafür, welches sein Begriff von Wissen ist. ${ }^{36}$

33 Vgl. Weinberg/Nichols/Stich 2008 [2001], S. 35f.

34 Beides Weinberg/Nichols/Stich 2008 [2001], S. 34.

35 Dieser Zusammenhang besteht nicht bei allen Begriffen im gleichen Umfang. Zwei Personen könnenvielleichtmit»gerecht«dasselbe meinen, ohne dieselben Anforderungen an das Vorliegen von Gerechtigkeit zu stellen. Und bestimmte Philosophinnen und Philosophen behaupten sogar, dass es Begriffe gibt, deren Anwendungskriterien man kennen kann, ohne zu wissen, was sie bedeuten. So argumentiert zum Beispiel Hans-Johann Glock dafür, dass es möglich ist, die Bedingungen dafür zu kennen, dass ein Vertrag gültig ist, ohne zu wissen, was es bedeutet, dass ein Vertrag gültig ist. Vgl. Glock 2003, S. 117 .

36 Interessanterweise kommt es auch in kritischen Auseinandersetzungen mit der Experimentellen Philosophie häufig zu Verwechslungen begrifflicher mit nicht-begrifflichen Aussagen (oder zumindest zu Formulierungen, die auf solche Verwechslungen hindeuten). So spricht Ralf Stoecker zum Beispiel von der Ansicht, dass man nur dasjenige absichtlich mache, was man beabsichtige (vgl. Stoecker 2014, S. 271; in der englischsprachigen Handlungstheorie wird diese Auffassung als »Simple View« bezeichnet. Vgl. Cushman/Mele 2008, S. 171), und Joseph Shieber schreibt: »the claim that no object has a surface that is red and green all over at the same time [...] is believed due to its intuitive plausibility« (Shieber 2010, S. 551, Kursivierung von J. S.). Triviale begriffliche Wahrheiten 
Ein weiterer Hinweis darauf, dass in den Abhandlungen von Vertreterinnen und Vertretern der Experimentellen Philosophie allenthalben übersehen wird, dass man es (wenigstens in der theoretischen Philosophie) im Wesentlichen mit begrifflichen Fragen zu tun hat, ist die verbreitete Rede von Alltagstheorien (folk theories). Dass Pat im Beispiel (iii) weiß, dass er ein Zebra sieht, und Milt in (iv) nicht absichtlich zwei Einsen würfelt (S. 259), ist weder Teil noch Konsequenz einer Theorie, sondern ergibt sich (sofern es stimmt) aus der Bedeutung der involvierten Ausdrücke. Die Idee, dass wir über Wissen, Intentionalität, moralische Verantwortlichkeit, freien Willen usw. Theorien oder Alltagstheorien haben, ist aber natürlich nicht charakteristisch für die Experimentelle Philosophie, sondern findet sich fast überall in der zeitgenössischen analytischen Philosophie. ${ }^{37}$

\section{Können wir aus dem Lehnstuhl beurteilen, welche Verwendungen sprachlicher Ausdrücke korrekt sind?}

\subsection{Die Bedeutung von »Wir würden sagen, dass ...»}

Wenden wir uns nun dem Einwand $\left(\mathrm{E}^{*}\right)$ zu. Dieser lautete: Wir können daraus, wie wir selbst ein Wort verwenden, nicht darauf schließen, wie andere Sprecherinnen und Sprecher es verwenden, und müssen, um zu Aussagen der Art »Wir würden sagen, dass das ein Fall von Wissen ist« berechtigt zu sein, empirische Studien durchführen.

Dieser Einwand beruht auf einem falschen Verständnis davon, was Ordinary Language-Philosophinnen und -Philosophen mit Aussagen vom Typ »Wir

wie die soeben erwähnte Aussage über Oberflächen, die ganz und gar rot oder ganz und gar grün sind, lassen keinen Raum für unterschiedliche Ansichten oder Grade der Plausibilität. Ich kann beispielsweise nicht der Ansicht sein, dass Junggesellen unverheiratet sind, da die Negation dieses Satzes, »einige Junggesellen sind verheiratet«, Unsinn ist. Und aus dem gleichen Grund ist es auch falsch, zu behaupten, es sei plausibel, dass Junggesellen unverheiratet sind. Anders verhält es sich mit den im vorliegenden Buch bereits mehrfach erwähnten synoptischen begrifflichen Wahrheiten. Synoptische begriffliche Wahrheiten sind nicht unmittelbar einsichtig, und deshalb können verschiedene Philosophinnen und Philosophen unterschiedliche Urteile in Bezug darauf fällen, ob sie wahr sind oder nicht. Ich wäre allerdings vorsichtig damit, in einem solchen Fall von verschiedenen Ansichten bzw. Meinungen zu sprechen: Wir haben es schließlich nicht mit dem zu tun, was wir als »Meinungsfragen« bezeichnen würden, und sobald die Zusammenhänge zwischen den relevanten Begriffen überblickt werden, lässt sich nicht mehr bestreiten, dass die betreffenden Sätze wahr sind: Eine der beiden »Meinungen« erweist sich also bei genauerer Betrachtung als inkonsistent.

Vgl. dazu auch das Kapitel 2 des vorliegenden Buchs. 
würden sagen, dass ...« meinen. Dass wir zum Beispiel nicht sagen würden, dass der Fall (iv) auf S. 259 ein Beispiel für absichtliches Handeln ist, ist keine Hypothese über das Verhalten einer Mehrheit der Sprachgemeinschaft, der die Sprecherin oder der Sprecher angehört, oder gar einer Mehrheit der Weltbevölkerung. »Wir würden sagen, dass der Fall (iv) kein Fall von absichtlichem Handeln ist « ist - entgegen dem, was man angesichts der Form des Satzes vermuten könnte - (1) nicht als hypothetisch aufzufassen und (2) eher normativ als deskriptiv zu verstehen.

$\mathrm{Zu}(1)$ : Die Idee ist nicht: Würde ein solcher Fall eintreten bzw. befände ich mich in einer derartigen Situation, dann würde ich sagen, dass es kein Fall von Absichtlichkeit sei. Sondern ich wende das fragliche Prädikat auf das Beispiel an, das mir als Gedankenexperiment gegeben ist, und sage hier und jetzt: Es ist kein Fall von absichtlichem Handeln. Wir könnten also ebenso gut schreiben: »Wir sagen nicht, dass das Beispiel (iv) ein Fall von absichtlichem Handeln ist.« Entsprechend kann eine Aussage der Art »Ich würde sagen, dass ...«, verstanden im hier relevanten Sinn, auch nicht widerlegt werden dadurch, dass ich zu einem späteren Zeitpunkt in einer Situation, die so geartet ist wie das Gedankenexperiment, behaupte, es sei ein Fall von absichtlichem Handeln. Sollte sich meine spontane Reaktion in der betreffenden Situation unterscheiden von meinem Lehnstuhl-Urteil, dann ist zwar die Rückfrage angebracht: »Was denn nun? Ist es ein Fall von absichtlichem Handeln oder nicht?«, aber der Reaktion in der realen Situation kommt keine Priorität zu gegenüber der Beurteilung aus dem Lehnstuhl bei der Beantwortung der Frage, was ich sagen würde. ${ }^{38}$

$\mathrm{Zu}(2)$ :Wenn ich den Satz äußere: »Wir würden sagen, dass der Fall (iv) kein Fall von absichtlichem Handeln ist«, dann bringe ich damit eine sprachliche Regel zum Ausdruck. Was ich meine, wenn ich den obigen Satz äußere, ist: Es wäre nicht korrekt, über jemanden, der sich in der Situation von Milt befindet, zu sagen, er habe absichtlich zwei Einsen gewürfelt. Und auch wenn wir dieses Urteil nicht wie im Kapitel 2 vorgeschlagen als Bekenntnis auffassen, sondern als Behauptung über die tatsächlich geltenden Regeln unserer geteilten Sprache, können wir nicht per Mehrheitsentscheid feststellen, ob es wahr ist oder nicht. Es ist möglich, dass eine Mehrheit der Sprecherinnen und Sprecher einer

$38 \quad$ Vgl. zu Abweichungen zwischen den Regeln für die Verwendung eines Wortes, die ein Sprecher »im Lehnstuhl« formuliert, und seiner tatsächlichen Verwendung dieses Wortes auch Kapitel 2, Abschnitt 3 des vorliegenden Buchs sowie Waismann 1976, S. 219. Welche Regel zum Gebrauch eines Wortes ein Sprecher tatsächlich befolgt, können wir Waismann zufolge am ehesten herausfinden, indem wir ihn auf die Abweichung aufmerksam machen und ihn fragen, welche Variante er als korrekt erachtet. 
Sprache einen bestimmten sprachlichen Ausdruck falsch verwendet. ${ }^{39}$ Ein Beispiel hierfür ist das Verb »sich wundern «. In meinem Umfeld verwenden immer mehr Leute dieses Verb im Sinne von »sich fragen «, entsprechend dem englischen »to wonder«. Sie äußern solche Sätze wie: »Ich wundere mich, ob mein Bruder heute Abend auch kommt. «Natürlich ist es gut möglich, dass diese Gebrauchsweise sich früher oder später durchsetzt und korrekt sein wird. Aber noch ist sie falsch, und das ändert sich auch nicht in dem Moment, in welchem mehr als 50 Prozent unserer Sprachgemeinschaft das Verb so verwenden. Ausschlaggebend dafür, dass es nach wie vor falsch ist, ist unter anderem der Umstand, dass Leute sich in dieser Verwendung üblicherweise korrigieren lassen. Weist man sie darauf hin, dass »sich wundern« eigentlich »erstaunt sein« bedeutet und dass sie es vermutlich wegen des englischen »to wonder« im Sinne von »sich fragen « verwenden, dann stimmen sie der Kritik zu.

Angesichts dessen, dass im »sich wundern«-Fall die oben erwähnte, falsche Gebrauchsweise um sich greift, wäre es allerdings natürlich irreführend, die Regel mit den Worten »Wir würden nicht so etwas sagen wie: >Ich wundere mich, ob mein Bruder heute Abend auch kommt « «u formulieren. Dies ist der Grund, weshalb ich weiter oben geschrieben habe, Äußerungen der Form »Wir würden sagen ... « seien eher normativ als deskriptiv, und nicht einfach, sie seien normativ: Wir verwenden diese Formulierung nur in Fällen, in welchen wir annehmen, dass die beschriebene Verwendung auch nach wie vor die geläufige Verwendung ist. Für die philosophische Argumentation relevant ist allerdings nur die normative Komponente, deshalb sehe ich nachfolgend von dieser Komplikation ab.

\subsection{Die Kompetenz zur Verwendung alltäglicher Ausdrücke}

Auch wenn »Wir würden nicht sagen, dass Milt im Beispiel (iv) absichtlich gehandelt hat « im Wesentlichen dasselbe bedeutet wie »Es wäre nicht korrekt, zu sagen, dass Milt im Beispiel (iv) absichtlich gehandelt hat«, stellt sich die Frage, wieso uns derartige Erkenntnisse aus dem Lehnstuhl zugänglich sein sollten. Im Zusammenhang mit dem »sich wundern«-Fall habe ich bereits geschrieben, dass dafür, welche sprachlichen Regeln gelten, u. a. ausschlaggebend ist, bei welcher Sorte von Verwendungen dieses Ausdrucks sich Sprecherinnen und Sprecher Korrekturen gefallen lassen. Allgemein spielen dafür, welche Gebrauchsweise eines Prädikats »ist F « richtig ist, diverse Aspekte eine Rolle:

- in welchen Situationen »ist $\mathrm{F}$ « und »ist nicht $\mathrm{F}$ « verwendet werden,

- wie dafür argumentiert wird, dass etwas $\mathrm{F}$ (oder nicht-F) ist bzw. wie entsprechende Behauptungen begründet werden, 
- wie die Bedeutung von »ist F « Kindern oder Sprecherinnen und Sprechern anderer Sprachen erklärt wird und,

- wie oben erwähnt, welche Verwendungen des Prädikats korrigiert werden und wie auf Korrekturen reagiert wird.

Jesse J. Prinz macht im Zusammenhang mit der Idee, dass unsere gängige Sprachpraxis bestimmt, welches unsere Begriffe sind, darauf aufmerksam, dass dies bedeuten würde, dass »introspektive Methoden « (und damit meint Prinz Lehnstuhl-Methoden im Allgemeinen) nicht dazu geeignet seien, sie zu erforschen. Wenn die von mir aufgezählten Parameter dafür ausschlaggebend sind, welches die geltenden Regeln zum Gebrauch sprachlicher Ausdrücke sind, dann müssen wir Prinz' Ansicht nach empirische Studien durchführen, in welchen beobachtet und dokumentiert wird, in welchen Situationen und auf welche Weise Sprecherinnen und Sprecher die Ausdrücke ihrer Sprache verwenden, ihre Äußerungen begründen, die Bedeutung der Ausdrücke erklären und so weiter. ${ }^{40}$

Urteile darüber, welche Regeln zur Verwendung sprachlicher Ausdrücke in Kraft sind, sind a posteriori; sie können nur empirisch gerechtfertigt werden. Wie die Wörter meiner Muttersprache verwendet werden, habe ich »durch Erfahrung « gelernt, es wurde mir von meinen Eltern und anderen Personen in meinem Umfeld beigebracht, aber dies hindert mich - besonders im Falle derjenigen Sorte von Wörtern, die in der Philosophie üblicherweise zur Diskussion stehen - nicht daran, heute, auf der Grundlage dieses empirisch erworbenen Wissens, im Lehnstuhl zu beurteilen, welche Gebrauchsweisen richtig oder falsch sind. »Absichtlich«, »wissen«, »geistig«, »freiwillig«, »sehen«, »Überzeugung «, »Eigenschaft«, »Bedeutung «, »wahr«, »begründen«, »Ursache«, »Person«, »versuchen«, »Wunsch« und »Handlung « werden im Alltag beispielsweise so häufig gebraucht, dass es keinen Zweifel daran gibt, dass ich sie richtig verwende.

Oswald Hanfling bezeichnet unsere Kenntnis der Regeln zur Verwendung solcher Ausdrücke als »participatory knowledge « ${ }^{41}$, durch »Mitmachen « erworbenes Wissen, bei dem wir und andere Leute um uns herum es schon längt gemerkt hätten, wenn wir nicht darüber verfügen würden. Dieses Wissen ist nicht propositional, wir können die Regeln, die wir befolgen, zumeist nicht vollständig formulieren. Wir verfügen aber über die Kompetenz (das Knowhow) zum Verwenden von »absichtlich «, »wissen « und so weiter, und es ist für uns in der Regel ein Kinderspiel, zu beurteilen, wie solche Wörter in einer beschriebenen Situation zu verwenden wären, oder zu begründen, weshalb wir in

40 Vgl. Prinz 2008, S. 193 f.

41 Hanfling 2000, S. 54. 
einem einzelnen Fall behauptet haben, dass $S$ weiß, dass $p$, oder dass $S$ absichtlich ge- $\phi$-t hat. Diese Behauptung sollte allerdings, wie in den nachfolgenden Abschnitten dargelegt wird, in verschiedenen Hinsichten qualifiziert werden.

\subsection{Die Möglichkeit von Fehlern}

Es ist nicht so, dass Ordinary Language-Philosophinnen und -Philosophen bei ihrer Beschreibung davon, wie wir einen sprachlichen Ausdruck verwenden, keine Fehler unterlaufen können. Berüchtigt ist zum Beispiel Gilbert Ryles Behauptung, dass wir das Wort »freiwillig « (voluntary) fast ausschließlich auf Handlungen anwenden, die nicht (hätten) begangen werden soll(t)en. ${ }^{42}$ Dies ist nicht der Fall, wir sagen zum Beispiel auch, dass sich jemand freiwillig für das Amt des Klassensprechers gemeldet hat, und es lassen sich beliebig viele ähnliche Fälle freiwilliger, aber nicht kritikwürdiger Handlungen konstruieren. $\mathrm{Zu}$ diesem Fehler von Ryle ist allerdings dreierlei zu bemerken:

\section{Bemerkung 1: Anwendung vs. Regel}

Ryle hat sich nicht einen Einzelfall vorgenommen und in Bezug auf diesen spezifischen Fall falsch beurteilt, wie er oder eine Gruppe von Sprecherinnen und Sprechern das Wort »freiwillig« darauf anwenden würde, sondern er hat eine Regel zur Verwendung von »freiwillig « formuliert, die nicht dem tatsächlichen Sprachgebrauch entspricht. Der Fehler passierte also nicht auf der Ebene der Beschreibung einer spezifischen Verwendungssituation oder im Kontext der Beurteilung eines konkreten Gedankenexperiments, wie es in der Experimentellen Philosophie angewendet wird.

Bemerkung 2: Bereinigung von Fehlern aus dem Lehnstuhl

Der Grund dafür, dass Ryle dieser Irrtum unterlaufen ist, dürfte gewesen sein, dass er bestimmte Fälle, wie etwa denjenigen des Klassensprechers, übersehen hat. Hätte er an den Klassensprecher- oder einen vergleichbaren Fall gedacht, hätte er vermutlich nicht behauptet, dass wir das Wort »freiwillig « nur auf unangebrachte Handlungen anwenden. Deshalb hätte ihm auch die Methode der Experimentellen Philosophie nicht weiterhelfen können: Mit einem Fall, den er selbst nicht im Auge hat, könnte Ryle auch keine Probandinnen und Probanden in einem Experiment konfrontieren. ${ }^{43}$ Und hätte er den Fall im Auge

42 Vgl. Ryle 1963, S. 67. Vgl. für eine Besprechung dieses Beispiels auch Hanfling 2000, S. $56 \mathrm{ff}$.

43 In dieser Hinsicht ist die Korpusanalyse der Experimentellen Philosophie überlegen. Hätte Ryle eine ganze Reihe tatsächlicher Verwendungen von »freiwillig« aus verschiedenen Äußerungskontexten durchgeschaut, hätte ihn dies vermutlich davor bewahrt, den oben erwähnten Fehler zu begehen. Welche der konsultierten Verwendungen 
gehabt, ist meines Erachtens, wie bereits erwähnt, anzunehmen, dass er ihn dann selbst als einen Fall von Freiwilligkeit klassifiziert hätte. Er hätte also auch dann keine Umfrage benötigt. ${ }^{44}$

Bemerkung 3: Konsequenzen idiosynkratischer Verwendungen Nehmen wir an, ich liege mit meiner obigen Einschätzung falsch und Ryle ist tatsächlich der einzige Sprecher, der das Wort »freiwillig« nicht auf den Klassensprecher-Fall und andere Fälle von Handlungen anwendet, gegen deren Ausführung nichts einzuwenden ist, und er würde daher dem Folgenden zustimmen:

(ix) Wir würden nicht sagen, dass der Klassensprecher-Fall ein Fall einer freiwilligen Handlung ist.

Damit würde er falsch liegen. Hier kommt allerdings ein weiterer wichtiger Punkt ins Spiel: Wenn (ix) Ryles reflektiertem Urteil entspricht, dann ist es ipso facto wahr, dass er selbst es als korrekt erachtet, dass Wort »freiwillig « wie in (ix) angegeben zu verwenden. Und solche Aussagen geben uns Aufschluss über die Regeln, die Ryle bei der Verwendung von »freiwillig « befolgt und damit über seinen Begriff der Freiwilligkeit. Und deshalb ist (ix) auch nicht philosophisch wertlos.

Gehen wir davon aus, Ryle äußert den Satz (ix) im Zusammenhang mit einem philosophischen Problem, das sich im Zusammenhang mit dem Begriff der Freiwilligkeit ergibt - zum Beispiel der Frage, ob es überhaupt möglich ist, aus freiem Willen zu handeln. Der hier vertretenen Auffassung zufolge können wir diese Frage auf der Grundlage einer Betrachtung davon beantworten, wie das Wort »freiwillig« für gewöhnlich verwendet wird. Es gibt nun zwei Optionen:Entweder es spielt für die Beantwortung der Frage nach der Möglichkeit freiwilliger Handlungen keine Rolle, ob dem Satz (ix) zugestimmt wird. In diesem Fall ist Ryles Lösung des Problems auch für eine Person hilfreich, die dem Satz (ix) nicht zustimmt. ${ }^{45}$ Oder es ist für das philosophische Problem, mit dem Ryle sich befasst, relevant, dass er dem Satz (ix) zustimmt. Dann kann ihm seine Erwägung immerhin helfen, seine eigene Verwirrung aufzulösen. Und noch wichtiger: Eine Abhandlung von einer anderen Person, die nicht denselben Begriff der Freiwilligkeit besitzt wie Ryle (und dem Satz (ix) nicht zustimmt), wäre dann nicht dafür geeignet, Ryles Problem zu lösen.

aus einem Textkorpus er als korrekt erachtet und für seine Regelformulierung berücksichtigt hätte, hätte aber auch dann in seinem eigenem Ermessen gelegen.

44 Vgl. dazu auch Sandis 2010, S. 187.

45 Vgl. dazu auch Fußnote 59. 
Dies bedeutet natürlich nicht, dass philosophische Probleme im Wesentlichen persönliche Probleme sind (eine Auffassung, die Hanfling Paul Grice zuschreibt ${ }^{46}$ ) oder dass wir uns in der Philosophie eigentlich immer nur darauf berufen, was wir selbst sagen (die Auffassung, die Alexander und Weinberg als »Intuitions-Solipsismus « bezeichnen ${ }^{47}$ ). Denn dass ein Philosoph ein häufig gebrauchtes Wort anders verwendet als andere Leute, mit denen er sich täglich austauscht, dürfte ein absoluter Ausnahmefall sein. Und wenn er weiß, dass seine Verwendung von der gewöhnlichen Verwendung abweicht, würde er seine Überlegungen zu seinem »persönlichen « Begriff sicherlich auch nicht publizieren. Dies aber nicht aus dem Grund, den Alexander und Weinberg gegen den Intuitions-Solipsismus anführen:

[I]f the appeal to intuitions is to make sense as part of an argumentative practice, then the evidentiary status of intuitions needs some foundation. In general, this foundation would have to arise either from the intuition's being shared with one's interlocutors, or from one's having some recognizable privileged authority with regard to the intuition in question. Neither type of foundation can be determined from a solipsistic perspective. Any argument that relied solely on the intuitions of the author of that argument would not have sufficient evidential strength and would have little hope of convincing anyone. ${ }^{48}$

Das Problem ist nicht, dass meine Argumente desto weniger Überzeugungskraft besitzen, je weniger andere Leute die für ein philosophisches Problem relevanten Ausdrücke so verwenden wie ich. Ich führe meine Art und Weise, ein Wort zu verwenden, nämlich nicht als Argument für eine philosophische These an (z. B. dafür, dass es möglich ist, aus freiem Willen zu handeln). Sondern meine Art und Weise, das Wort »freiwillig « zu verwenden, legt fest, was meine philosophische Frage bedeutet bzw. worin mein philosophisches Problem besteht, und philosophische Fragen und Probleme sollten natürlich unter Berücksichtigung ihrer Bedeutung beziehungsweise ihres Gehalts beantwortet resp. gelöst werden. Andere Leute teilen aber meine philosophischen Probleme nur in dem Maße, in dem sie meine Begriffe teilen, und deshalb ist beispielsweise die Beantwortung meiner Frage, ob es freiwillige Handlungen gibt, für sie auch nur in dem Maße aufschlussreich, in welchem sie mit »freiwillig « dasselbe meinen wie ich.

Ich habe weiter oben geschrieben, dass es nur sehr selten vorkommt, dass eine Person einen häufig verwendeten Ausdruck anders gebraucht als die Leute um sie herum. Deshalb ist meine Behauptung, dass beispielsweise

$46 \quad$ Vgl. Hanfling 2000, S. 59.

47 Vgl. Alexander/Weinberg 2007, S. 57.

48 Alexander/Weinberg 2007, S. 57f. 
Ryles Ausführungen zur Freiwilligkeit auch dann nicht philosophisch wertlos wären, wenn er dem Satz (ix) zustimmen würde, nicht von großer praktischer Relevanz. Was ich über die Begriffe von Einzelpersonen gesagt habe, gilt aber erst recht für die Begriffe von Gruppen von Personen. Sollte es also der Fall sein, dass das Wort »freiwillig « in der Schweiz anders verwendet wird als in Deutschland oder Österreich oder von $50 \%$ der deutschsprachigen Bevölkerung anders als von den anderen $50 \%$, dann tut dies dem Wert - und vor allem dem Wahrheitsgehalt - einer philosophischen Abhandlung keinen Abbruch. ${ }^{49}$ Es sollte deshalb auch nicht angenommen werden, dass sich »Wir« in »Wir würden nicht sagen, dass Milt im Beispiel (iv) auf S. 259 absichtlich zwei Einsen gewürfelt hat « auf alle Sprecherinnen und Sprecher des Deutschen bezieht. Was mit diesem Satz gemeint ist, ist, dass es wenigstens einer Regel zur Verwendung von »absichtlich « entspricht, zu sagen, dass Milt nicht absichtlich zwei Einsen gewürfelt hat, und dass diese nicht nur von mir, sondern von einer ganzen Gruppe von Personen befolgt wird. ${ }^{50}$

\subsection{Schwierige Fälle}

Kommen wir nun zurück zur obigen Behauptung, wir könnten aus dem Lehnstuhl beurteilen, ob ein beschriebener Fall unter ein Prädikat wie »verfügt über Wissen« oder »hat absichtlich gehandelt« falle oder nicht.

Tatsächlich gibt es unter den Gedankenexperimenten, die Philosophinnen und Philosophen konstruiert haben, Fälle, in welchen es nicht einfach ist, zu entscheiden, ob sie Beispiele für Wissen, Absichtlichkeit usw. sind oder nicht. Ein Beispiel dafür ist das berühmte Gedankenexperiment von Joshua Knobe betreffend die Nebeneffekte unserer Handlungen:

The vice-president of a company went to the chairman of the board and said, "We are thinking of starting a new program. It will help us increase profits, but it will also harm the environment."

The chairman of the board answered, "I don't care at all about harming the environment. I just want to make as much profit as I can. Let's start the new program."

They started the new program. Sure enough, the environment was harmed.

Now ask yourself: Did the chairman of the board intentionally harm the environment? ${ }^{51}$

49 Vorausgesetzt, natürlich, dass die Abhandlung nicht explizit für sich beansprucht, die Regeln des Deutschen darzulegen.

5o Vgl. dazu auch Fußnote 75 des vorliegenden Kapitels.

51 Knobe 2006, S. 205f. Zu den betreffenden Befragungen gibt es von Knobe auch eine ältere Publikation, nämlich Knobe 2003. 
Ich bin nicht sicher, ob ich sagen würde, dass der Vorstandsvorsitzende die Umwelt absichtlich geschädigt hat, oder nicht. Bedeutet dies, dass mir die Regeln zur Verwendung von »absichtlich « nicht ganz klar sind bzw. dass meine Kompetenz zur Verwendung dieses Wortes unzureichend ist? Dies ist eine Möglichkeit, obwohl es im Falle eines so geläufigen Wortes wie »absichtlich « und einer Muttersprachlerin erstaunlich wäre. Wahrscheinlicher ist, dass ich mich (a) in einem Zustand von vorübergehender Verwirrtheit befinde, beispielsweise weil ich in der letzten Zeit so viele handlungstheoretische Texte gelesen habe, dass ich aus den Augen verloren habe, wie ich »absichtlich« normalerweise verwende, oder (b) dass die etablierten Regeln zum Gebrauch von »absichtlich « gar nicht festlegen, ob der Vorstandsvorsitzende im obigen Beispiel die Natur absichtlich geschädigt hat oder nicht. So schreibt zum Beispiel Ralf Stoecker, es sei weder natürlich, zu sagen, der Vorstandsvorsitzende habe die Umweltschädigung absichtlich herbeigeführt, noch, er habe sie unabsichtlich herbeigeführt. Was wir stattdessen zum Beispiel sagen würden, sei, dass er sie bewusst oder wissentlich herbeigeführt habe oder dass er die Schädigung in Kauf genommen habe. ${ }^{52}$

Konfrontiert mit dem Beispiel von Knobe wäre es für mich sinnvoll, mich bei einigen anderen Personen zu erkundigen, wie sie den Fall beschreiben würden. Wenn sie alle auch unsicher sind, spricht dies für die oben erwähnte Option (b). Sagen sie jedoch alle ohne zu zögern, der Vorstandsvorsitzende habe die Umwelt absichtlich geschädigt, ${ }^{53}$ dann wäre es für mich wichtig, wie sie ihre Behauptung begründen. Es ist denkbar, dass sie mir deutlich machen können, dass das Beispiel in relevanter Hinsicht gleich geartet ist wie bestimmte andere Fälle, in welchen es auch für mich offensichtlich ist, dass sie Fälle absichtlicher Handlungen sind. In diesem Fall würde mir klarwerden, dass die Option (a) eingetreten ist und ich mir einfach einen Moment lang nicht darüber im Klaren war, wie ich das Wort »absichtlich« verwende. Würden andere Leute einstimmig dafür plädieren, dass der Vorstandsvorsitzende absichtlich gehandelt hat, ohne mir dies begreiflich machen zu können, dann hätten wir es wohl mit einem Fall zu tun, in welchem es eine etablierte Regel gibt, die ich nicht kenne. Was ich mit dieser Erkenntnis anfangen sollte, ist allerdings nicht vollständig klar. Es wäre vielleicht vernünftig, das Wort »absichtlich« in Zukunft ebenfalls dieser Regel entsprechend zu verwenden, aber damit hätte ich nichts über die Absichtlichkeit von Nebeneffekten gelernt, was ich vorher

52 Vgl. Stoecker 2014, S. 264, 276, Fußnote 33.

53 Ich bespreche hier nur diese Reaktion, da dies offenbar die Antwort ist, die tatsächlich meistens gegeben wird. Vgl. die weiter unten im vorliegenden Abschnitt besprochenen Umfrage-Resultate. 
noch nicht wusste (denn in welchem Verhältnis z. B. der Vorstandsvorsitzende zur Herbeiführung der Umweltschädigung steht, war mir vorher bereits klar).

Welche Rolle könnte die Befragung einer großen Zahl von Sprecherinnen und Sprechern in einem Szenario spielen, in welchem ich nicht sicher bin, wie (bzw. ob) ich einen Ausdruck auf einen beschriebenen Fall anwenden würde? Die Studie von Knobe hat ergeben, dass $82 \%$ der Leute sagen, dass der Vorstandsvorsitzende im oben geschilderten Fall die Umwelt absichtlich geschädigt hat. ${ }^{54}$ Tatsächlich wurden die Probandinnen und Probanden in seinem Experiment auch zu den Gründen für ihre Antwort befragt, ${ }^{55}$ so dass die gesamten Umfrage-Ergebnisse (im Gegensatz zur oben genannten Prozentzahl allein) denselben Nutzen für mich haben könnten wie die von mir vorgeschlagene Diskussion mit Freunden oder Berufskolleginnen. Wichtig ist aber, hier zu sehen, dass der Nutzen der Umfrage nicht (oder jedenfalls nicht im von den Vertreterinnen und Vertretern der Experimentellen Philosophie behaupteten Umfang ${ }^{56}$ ) über den Nutzen eines Gesprächs mit einer »nichtrepräsentativen « Zahl kompetenter Sprecherinnen und Sprecher hinausgeht. Selbst wenn wir annehmen, dass die $82 \%$, die gesagt haben, der Vorstandsvorsitzende habe die Umwelt absichtlich geschädigt, den Ausdruck »absichtlich« tatsächlich so verwenden, wie sie es in der Umfrage angegeben haben (was aus Gründen zu bezweifeln ist, die ich weiter unten im Abschnitt 3.6 bespreche), ist nicht klar, ob die anderen $18 \%$ sich über ihren Sprachgebrauch geirrt haben oder es einfach zwei verschiedene Verwendungen des Ausdrucks gibt und damit zwei (evt. nur geringfügig) verschiedene Absichtlichkeits-Begriffe. Und um mich daran zu erinnern, wie ich einen Ausdruck verwende, reicht eine einzige Person, die mich beispielsweise auf eine bestimmte Analogie aufmerksam macht. Die Kategorie der statistischen Signifikanz spielt dabei keine Rolle.

54 Vgl. Knobe 2006, S. 206. Ersetzt man jedoch im Beispiel jedes Vorkommnis von »die Umwelt schädigen « durch »der Umwelt helfen« und fragt die Probandinnen und Probanden, ob der Vorstandsvorsitzende der Umwelt absichtlich geholfen habe, so wird die Frage nur von $23 \%$ bejaht (wobei jede Probandin und jeder Proband entweder nur zum Schädigungs-Fall oder nur zum Hilfe-Fall befragt wurde) (vgl. Knobe 2006, S. 206).

55 Vgl. dazu auch Austin 1956, S. 7: Wenn wir in der Philosophie die Ordinary LanguageMethode anwenden, untersuchen wir Austin zufolge, »what we should say when, and so why and what we should mean by it« (Hervorhebung von mir verändert).

$5^{6}$ Eine gute Befragung kann natürlich bestimmte Vorteile gegenüber einem Gespräch zwischen Tür und Angel mit einem Kollegen oder einer Kollegin haben. Zum Beispiel dass die Probandinnen und Probanden ihre Aufmerksamkeit ganz auf die gestellte Frage lenken, nicht zugunsten (oder zuungunsten) einer allenfalls im informellen Gespräch von der Fragestellerin suggerierten Antwort voreingenommen sind usw. 


\subsection{Geübtheit im Umgang mit Gettier-Fällen?}

Meine obige Behauptung, bei Wörtern, die so häufig verwendet würden wie »wissen« oder »absichtlich«, gebe es keine Zweifel daran, dass wir alle wissen, wie sie richtig verwendet werden, könnte neben den in den vorangehenden Abschnitten behandelten Bedenken auch aufgrund der folgenden Überlegung infrage gestellt werden: Es mag sein, dass wir jeden Tag mitbekommen, wie diese Wörter auf gewöhnliche Fälle angewendet werden, aber woher sollten wir wissen, welches ihre korrekte Anwendung auf außergewöhnliche Fälle ist und wie etwa der Ausdruck »wissen« auf einen Gettier-Fall anzuwenden ist? Ich gerate, wie vermutlich auch die meisten anderen Leute, äußerst selten in Situationen, in welchen

(a) eine Person $S$ die wahre Überzeugung hat, dass $p$,

(b) die auf einer falschen Überzeugung beruht, dass $q$, und

(c) in welcher sich jemand, der (wie ich auch) über (a) und (b) informiert ist, dazu veranlasst sieht, zu entscheiden, ob $S$ wisse, dass $p$, oder nicht.

Woher sollte also meine Übung in der Anwendung des Ausdrucks »wissen « auf Gettier-Fälle herrühren? ${ }^{57}$

$\mathrm{Zu}$ dieser Frage ist zunächst einmal zu bemerken, dass wir nicht für jeden spezifischen Einzelfall separat lernen müssen, unter welche Begriffe er fällt oder nicht fällt. Offenbar hat Edmund Gettier, als er 1963 seine beiden Gedankenexperimente zur Widerlegung der Justified-True-Belief-Analyse von Wissen präsentiert hat, angenommen, dass wir alle spontan zustimmen, dass diese Fälle keine Fälle von Wissen sind. Und tatsächlich lassen sich die meisten Leute $^{58}$ von Gettiers Argumentation überzeugen. Dies spricht dafür, dass uns die alltägliche Praxis der Verwendung des Ausdrucks »wissen«, zu der, wie oben erwähnt, auch die Begründungen für Wissenszuschreibungen oder für das Absprechen von Wissen gehören, Regeln an die Hand geben, die auch festlegen, dass in Gettier-Fällen kein Wissen vorliegt.

Wäre es hingegen so, dass wir im Falle von Gettier-Fällen nachhaltig unentschlossen wären, ob wir Wissen zuschreiben sollen oder nicht, dann würde wieder die Argumentation aus dem Abschnitt 3.4 weiter oben Anwendung finden: Es ist möglich, dass für meinen Wissensbegriff gar nicht festgelegt ist, ob Gettier-Fälle unter ihn fallen oder nicht. Wie eine Mehrheit kompetenter Sprecherinnen und Sprecher Gettier-Fälle beurteilt, ist außerdem erstens prinzipiell kein Kriterium dafür, welche Verwendung richtig oder falsch ist, und zweitens besteht immer die Möglichkeit, dass ich meinen Wissensbegriff

57 Vgl. dazu auch das Kapitel 3 des vorliegenden Buchs.

$5^{8}$ Zumindest die meisten Westlerinnen und Westler; vgl. die Ausführungen zum Einwand $\left(\mathrm{E}^{*}{ }^{*}\right)$ weiter unten. 
nur mit einer Minderheit anderer Sprecherinnen und Sprecher teile, was der Relevanz einer Analyse meines eigenen Begriffs für die Lösung eines sich für mich ergebenden philosophischen Problems keinen Abbruch tut, aber großangelegte Befragungen als Methode dafür ausschließt. ${ }^{59}$

\subsection{Erklärung der Diversität}

Ein letzter naheliegender Einwand gegen meine Behauptung, wir könnten aus dem Lehnstuhl beurteilen, wie ein Ausdruck korrekterweise auf einen beschriebenen Fall angewendet werde, dürfte durch die vorangehenden Absätze schon teilweise entkräftet worden sein. Der Einwand lautet: Wenn wir alle wissen, wie beispielsweise der Ausdruck »wissen« zu verwenden ist - wie erklären wir dann die von Vertreterinnen und Vertretern der Experimentellen Philosophie nachgewiesene Diversität in den Reaktionen auf Gedankenexperimente?

DerUmstand, dass in den Befragungen durch Vertreterinnen undVertreter der Experimentellen Philosophie auch dann unterschiedliche Antworten gegeben werden, wenn die befragten Personen allesamt kompetente Sprecherinnen und Sprecher sind, ist auf verschiedene Faktoren zurückzuführen:

\section{Faktor 1: Problematische Fragestellungen}

Erstens gibt es teilweise Unklarheiten, Mehrdeutigkeiten oder andere Probleme im Zusammenhang mit den in den Befragungen verwendeten Gedankenexperimenten und den darauf bezogenen Fragen. Teilweise dürften die Antworten der Probandinnen und Probanden beeinflusst sein durch unterschiedliche, durch die Fallbeschreibungen nicht ausgeschlossene

59 Im Übrigen sollte die Relevanz von Gettier-Fällen und ähnlich exotischen Fällen für die Epistemologie nicht überschätzt werden. Im Kontext der Suche nach notwendigen und hinreichenden Bedingungen für das Vorliegen von Wissen haben solche Fälle sicher einen Nutzen, aber von diesem Projekt haben sich viele Vertreterinnen und Vertreter der Ordinary Language-Philosophie abgewendet, da sie es für aussichtslos und/oder nicht besonders interessant erachten. Mit der Frage, was Wissen ist, kann man sich auch gewinnbringend befassen, ohne sich der Suche nach notwendigen und hinreichenden Bedingungen zu verschreiben, vgl. z. B. Hanfling 2000, Kap. 6. Und für die Behandlung zahlreicher anderer philosophischer Probleme, die sich im Zusammenhang mit Wissen ergeben - z. B. für das Problem des Skeptizismus oder für Moores Paradox - spielt es unter Umständen gar keine Rolle, ob wir Gettier-Fälle als Fälle von Wissen auffassen oder nicht. Ein und dieselbe Behandlung des Skeptizismus oder ein und dieselbe Auflösung von Moores Paradox kann gleichermaßen hilfreich sein für Personen, die Gettier-Fälle als Fälle von Wissen beschreiben, und für Personen, die dies nicht tun. 
Hintergrund- oder Zusatzannahmen. ${ }^{60}$ Wie die Frage zum Zebra-Fall auf S. 259 des vorliegenden Kapitels beantwortet wird, hängt zum Beispiel unter Umständen davon ab, ob angenommen wird, dass es für Zoos irgendeinen Anreiz gibt, anstelle eines Zebras ein bemaltes Maultier in ein Gehege zu setzen, und ob den Mitarbeiterinnen und Mitarbeitern zuzutrauen ist, dass sie so etwas machen.

Ein Beispiel für eine Ambiguität in der von Vertreterinnen und Vertretern der Experimentellen Philosophie verwendeten Fragestellung liefert Daniel Cohnitz: Die Frage zum Gödel-Fall, der auf S. 26o des vorliegenden Kapitels angeführt wurde, sei ambig zwischen »Wen meint John, wenn er den Namen $>$ Gödel verwendet? « und »Was (bzw. wer) ist das tatsächliche Bezugsobjekt des Namens >Gödel«?«, d. h. zwischen Sprecher-Referenz und semantischer Referenz. ${ }^{61}$ Zum Teil sind die verwendeten Gedankenexperimente auch einfach so kompliziert, dass wir uns nicht darauf verlassen können, dass die Probandinnen und Probanden den zu beurteilenden Fall verstehen und überblicken. ${ }^{62}$ Eine weitere Schwierigkeit aus derselben Kategorie sind irreführende Fragestellungen. So erscheint es mir zum Beispiel, wie bereits im Kapitel 3 des vorliegenden Buchs angemerkt, problematisch, dass Weinberg, Nichols und Stich nach ihren Schilderungen der einzelnen Gettier-, Truetempund anderen epistemologischen Fallbeispiele jeweils gefragt haben: »Weiß die betreffende Person wirklich, dass das-und-das der Fall ist, oder glaubt sie es nur? «. ${ }^{63}$ Dass jemand nur glaubt, dass $p$, würde ich in der Regel nur dann behaupten, wenn $p$ nicht der Fall ist oder die betreffende Person nicht sicher ist, ob $p$ der Fall ist. Für die Beurteilung der von Weinberg, Nichols und Stich verwendeten Fälle würde ich deshalb die Frage »Weiß die Person, dass $p$, oder weiß sie es nicht? « bevorzugen. Tatsächlich hat Simon Cullen festgestellt, dass die Antworten anders ausfallen, wenn die Fragestellung wie von mir vorgeschlagen angepasst wird. ${ }^{64}$

60 Vgl. Sosa 2009, S. 107f., Cushman/Mele 2008, S. 171, 179f., Grundmann/Horvath/Kipper 2014, S. 18f., Brendel 2014, S. 199-201, 206.

61 Vgl. Cohnitz 2014, S. 242f., 251, 255.

62 Dies gilt zum Beispiel für die Fallbeschreibungen, die Nichols und Knobe in »Moral Responsibility and Determinism« verwendet haben. Vgl. Nichols/Knobe 2007, S. 668-671, 675-677.

63 Vgl. Weinberg/Nichols/Stich 2008 [2001], S. 26-34. Z. B. »Does Mike really know that the animal is a zebra, or does he only believe that it is?« (S. 32).

64 Vgl. Cullen 2010, S. 288f. Cullen störte an der ursprünglichen Formulierung allerdings hauptsächlich, dass die erste Antwortoption statt $» S$ weiß, dass $p$ « lautete: $S$ weiß wirklich, dass $p \ll$ (really knows). 
Faktor 2: Unterbestimmtheit

Ein zweiter Ansatz zur Erklärung der Unterschiede in den von Vertreterinnen und Vertretern der Experimentellen Philosophie erhobenen Reaktionen auf Gedankenexperimente hängt mit dem Punkt zusammen, den ich im Abschnitt 3.4 des vorliegenden Kapitels besprochen habe: Dass in der Experimentellen Philosophie (wie auch in der zeitgenössischen analytischen Philosophie im Allgemeinen) auf Beispiele zurückgegriffen wird, im Falle derer es schwierig ist, zu beurteilen, ob sie unter den zur Diskussion stehenden Begriff fallen oder nicht, ist eher die Regel als die Ausnahme. In der Tat wäre es interessant, zu sehen, was die Vertreterinnen und Vertreter der Experimentellen Philosophie - sowohl der Experimentellen Analyse als auch des Experimentellen Restriktionismus - dazu sagen würden, wenn die Reaktionen von Probandinnen und Probanden in Bezug auf solche Fälle auseinandergehen würden, die ganz eindeutig Fälle von Wissen oder von Absichtlichkeit sind. Unter den Gedankenexperimenten von Fiery Cushman und Alfred Mele, von denen auch das Beispiel (iv) auf S. 259 stammt, gibt es das eine oder andere, in welchem es eigentlich keine Zweifel daran geben dürfte, welches die richtige Antwort ist. Zum Beispiel das folgende:

Jen sees some bothersome weeds growing next to her driveway and wants to eliminate them. She decides to go to the hardware store to buy weed spray. As she pulls out of her garage, the wheel slips in her hand and she drives off to the side of the driveway. All the weeds are crushed and killed under the car. With the weeds eliminated, Jen doesn't need to go to the hardware store. Did Jen intentionally eliminate the weeds? ${ }^{65}$

Nehmen wir an, es würden 6o\% der Teilnehmerinnen und Teilnehmer einer Umfrage sagen, dies sei ein Fall von absichtlichem Handeln. Würde dies zeigen, dass ich mich irre, wenn ich meine, dass Jen das Unkraut nicht absichtlich beseitigt hat? Würde es bedeuten, dass ich meine »Absichtlichkeits-Theorie« hinterfragen sollte? Ich glaube nicht, dass in diesem Fall irgendjemand dazu geneigt wäre, etwas anderes zu behaupten, als dass 6o\% der Probandinnen und Probanden entweder das Beispiel nicht richtig gelesen oder verstanden haben oder unter »absichtlich« etwas anderes verstehen als wir. Aber wenn die Diversität in den Antworten in diesem Fall so beurteilt würde, wieso werden dann aus der Diversität in Gettier-Fällen und anderen exotischen Fällen vonseiten der Experimentellen Philosophie ganz andere Schlussfolgerungen gezogen?

65 Cushman/Mele 2008, S. 181. 
Dass die Reaktionen auf die schwierigen Fälle, mit welchen sich die Vertreterinnen und Vertreter der Experimentellen Philosophie üblicherweise auseinandersetzen, so stark auseinandergehen, liegt jedenfalls meines Erachtens daran, dass für diese Fälle häufig überhaupt nicht bestimmt ist, wie ein bestimmtes Prädikat auf sie anzuwenden ist. Ich habe diese Möglichkeit weiter oben bereits im Zusammenhang mit Knobes Frage nach der Absichtlichkeit der Herbeiführung von Nebeneffekten erwähnt: Es ist schwierig, zu entscheiden, ob im Vorstandsvorsitzenden-Fall (S. 272) den Gemeinsamkeiten mit einem paradigmatischen Fall von Absichtlichkeit eine größere Relevanz zukommt als den Abweichungen von einem paradigmatischen Fall. Die Probandinnen und Probanden einer entsprechenden Umfrage werden sich jedoch typischerweise dazu genötigt fühlen, zu entscheiden, ob wir es im betreffenden Fall mit einem Fall von Absichtlichkeit zu tun haben oder nicht. Und durch die Fragestellung wird suggeriert, dass es entweder wahr ist, dass der Nebeneffekt absichtlich herbeigeführt wurde, oder falsch. Die Antwort, dass die Behauptung, es handle sich um einen Fall von Absichtlichkeit, ebenso wenig zum Vorstandsvorsitzenden-Fall passt wie ihre Negation, wird den Probandinnen und Probanden nicht zur Auswahl gestellt (und würde ihnen vielleicht aufgrund von Vorurteilen der Art, wie sie in Kapitel 1, Abschnitt 3.3 des vorliegenden Buchs beschrieben werden, auch gar nicht plausibel erscheinen).

\section{Faktor 3: Unterschiedliche Begriffe}

Sicherlich haben die Vertreterinnen und Vertreter der Experimentellen Philosophie auch Befragungen durchgeführt, im Falle derer die Unterschiede in den Antworten weder durch problematische Fragestellungen noch durch Unterbestimmtheit erklärt werden können. In solchen Fällen kommt eine dritte Begründungsstrategie ins Spiel: Die Unterschiede in den Reaktionen auf die Gedankenexperimente sind darauf zurückzuführen, dass die Probandinnen und Probanden über unterschiedliche Begriffe verfügen und z. B. unter »Wissen « oder »absichtlich « nicht dasselbe verstehen.

Gegen die Idee, dass sich in ihren Befragungen ganz einfach zeige, dass es verschiedene Begriffe von Wissen, Absichtlichkeit usw. gebe, haben die Vertreterinnen und Vertreter der Experimentellen Philosophie einige Einwände vorgebracht, auf die nachfolgend reagiert werden soll.

Grundmann, Horvath und Kipper machen darauf aufmerksam, dass die Berufung auf unterschiedliche Begriffe nicht erklären kann, weshalb die Antworten der Probandinnen und Probanden mit der Reihenfolge der vorgetragenen Beispiele variieren. ${ }^{66}$ Diese Varianz ist meines Erachtens jedoch 
offensichtlich mit Suggestion zu erklären: Wenn ich einer Testperson beispielsweise zuerst einen klaren Fall von Wissen präsentiere und dann eine leicht veränderte Version davon, dann wird sie dazu geneigt sein, anzunehmen, dass das zweite Beispiel ein Fall von Nichtwissen sei (und umgekehrt).

Des Weiteren schreiben Grundmann, Horvath und Kipper:

Zudem droht hier die inflationäre Zuschreibung von begrifflichen Unterschieden auf der Grundlage von oft nur minimalen Unterschieden in der Bewertung von wenigen nichtalltäglichen Fällen. ${ }^{67}$

Dieses Argument findet sich auch bei Weinberg, Nichols und Stich, die die Idee, dass es verschiedene Bedeutungen von »Wissen« gibt, auf der Grundlage der Beobachtung zurückweisen, dass die Probandinnen und Probanden der von ihnen durchgeführten Studien die allermeisten Fälle gleich beurteilen. Dies lege nahe, dass die verschiedenen Kulturen, sozioökonomischen Gruppen, Geschlechter usw. prinzipiell durchaus eine Alltagstheorie des Wissens miteinander teilen. ${ }^{68}$

Diesem Einwand ist Folgendes entgegenzuhalten: Wir können einräumen, dass wir in dem Maße unterschiedliche Begriffe besitzen, in welchem unsere Verwendung des Ausdrucks »wissen« unterschiedlichen Bedingungen unterliegt, ${ }^{69}$ und trotzdem weiterhin dem Umstand Rechnung tragen, dass wir mit »wissen « fast dasselbe meinen. Wenn mein Wissensbegriff sich vom Wissensbegriff meines Nachbarn lediglich darin unterscheidet, dass mein Nachbar im Gegensatz zu mir Gettier-Fälle als Fälle von Wissen klassifiziert, dann überschneiden sich unsere Wissensbegriffe in signifikantem Maße. Und da es die Möglichkeit einer solchen Begriffsüberschneidung gibt, folgt daraus, dass wir nicht den exakt gleichen Wissensbegriff haben - entgegen dem, was Mallon, Machery, Nichols und Stich befürchten ${ }^{70}$ - auch nicht, dass wir pausenlos aneinander vorbeireden. Entsprechend können wir auch etwas über den Geist lernen, wenn wir englische Bücher und Artikel zum Thema mind lesen - obwohl es diverse Unterschiede zwischen dem Gebrauch von »Geist«

67 Grundmann/Horvath/Kipper 2014, S. 20.

68 Vgl. Weinberg/Nichols/Stich 2008 [2001], S. 35f. Vgl. für ein ähnliches Argument auch Baz 2012, S. 10of. und den Abschnitt 4.3.1 des Kapitels 5 des vorliegenden Buchs.

69 Dass Weinberg, Nichols und Stich diesen Zusammenhang zwischen einem Begriff und den Anwendungsbedingungen des entsprechenden Wortes als weniger eng auffassen, habe ich bereits weiter oben kritisiert. Vgl. S. 264 des vorliegenden Kapitels.

Vgl. Mallon/Machery/Nichols/Stich 2009, S. 348-351. 
im Deutschen und demjenigen von »mind « im Englischen gibt und die beiden Begriffe mithin nicht identisch sind. ${ }^{71}$

\section{$4 \quad$ Wieso sollten wir unsere eigenen Begriffe privilegiert behandeln?}

Die Auffassung, dass es verschiedene Begriffe des Wissens, der Absichtlichkeit, der Freiwilligkeit usw. geben kann, leitet uns direkt zum zweiten der auf S. 258 formulierten Einwände, dem Einwand $\left(\mathrm{E2}^{*}\right)$ über. Dieser lautete: Wie sich herausgestellt hat, verwenden verschiedene Personen beispielsweise das Wort »wissen « (oder seine Entsprechungen in anderen Sprachen) unterschiedlich, und es gibt keinen Grund zur Annahme, dass unsere Weise, das Wort zu verwenden, eine größere Aussagekraft in Bezug darauf hat, was Wissen tatsächlich ist, als die anderen Verwendungsweisen. Tatsachen in Bezug darauf, was wir sagen würden, sind also nicht als philosophische Evidenz geeignet.

Gehen wir davon aus, dass Personen, die ostasiatischen Ursprungs sind, aber in den USA studieren und fließend Englisch sprechen, tatsächlich viel stärker als Westlerinnen und Westler dazu neigen, über Gettier-Fälle zu sagen, sie seien Fälle von »knowledge « - und zwar ohne dass sie diese Fälle missverstehen oder andere Zusatzannahmen in Bezug auf die betreffenden Szenarien voraussetzen als die befragten Westlerinnen und Westler. ${ }^{72}$ Und nehmen wir zusätzlich an, es gebe in den ostasiatischen Sprachen jeweils ein Wort, das normalerweise (etwa in Übertragungen literarischer Werke ins Japanische, Mandarin usw.) als Übersetzung von »to know « verwendet wird und dessen gängiger Gebrauch sich vom gängigen Gebrauch von »to know « nur darin unterscheidet, dass es auch auf Gettier-Fälle Anwendung findet. Wieso sollten wir dann annehmen, dass unsere Verwendung von »know « besser ist als diejenige der Ostasiatinnen und Ostasiaten? Beziehungsweise: Wieso sollte unsere Verwendung von »know « besser dazu geeignet sein, uns Aufschluss darüber zu geben, was Wissen ist, als der Gebrauch der Entsprechung dieses Wortes in ostasiatischen Sprachen? Solche Fragen werden in den Texten von Vertreterinnen und Vertretern der Experimentellen Philosophie immer wieder aufgeworfen. So schreiben Knobe und Nichols zum Beispiel in ihrem »Experimental Philosophy Manifesto «:

71 Vgl. zur Überschneidung der Bedeutung von Wörtern unterschiedlicher Sprachen auch Waismann 1976, S. 253.

72 Vgl. Weinberg/Nichols/Stich 2008 [2001], S. 28-30. 
If I find out that my philosophical intuitions are a product of my cultural upbringing, then, since it's in some sense an accident that I had the cultural upbringing that I did, I am forced to wonder whether my intuitions are superior at tracking the nature of the world, the mind, and the good. [...] And just as some Christian children come to think that there's no rational basis for preferring Christian to Hindu beliefs, we too might come to think that there's no rational basis for preferring Western philosophical notions to Eastern ones. ${ }^{73}$

Und Nadelhoffer und Nahmias sind der Meinung, wenn wir uns in der Philosophie beispielsweise auf unsere eigene Beurteilung von Gettier-Fällen stützen und uns aufgrund unserer eigenen »Intuitionen« darauf verlassen, dass Gettier-Fälle keine Fälle von Wissen sind, dann entspreche dies im Lichte der Diversität in den Reaktionen der Angehörigen unterschiedlicher Kulturen auf solche Fälle einer ungerechtfertigten Privilegierung unserer eigenen Kultur:

[I]f our intuitions about a particular topic vary cross-culturally or socio-economically and we don't have independent grounds for privileging our own intuitions to those of others, these particular intuitions will be insufficient for philosophical theory building.

The worry expressed by ERs [i. e. Experimental Restrictionists] is that in light of the gathering evidence concerning the wide-scale diversity of intuitions, intuition-driven philosophy ends up being both epistemologically xenophobic and intuitionally question-begging. For instance, Weinberg, Stich, and Nichols have run studies that suggest Westerners apply the concept of knowledge differently than East Asians [...] The results from these studies suggest that without a (culturally neutral) error theory to explain why one culture's intuitions are mistaken, these cross-cultural differences put pressure on both the evidentiary status of intuitions and on the ability of reflective equilibrium to help us separate the good intuitions from the bad ones. ${ }^{74}$

Diese Argumentation mag vielleicht angemessen sein, wenn wir es mit der Frage zu tun haben, ob uns unsere moralischen Intuitionen darüber Aufschluss geben können, was richtig oder falsch ist. Im Zusammenhang mit einem Begriff wie demjenigen des Wissens und der Frage, was Wissen ist, ist sie jedoch unpassend. Wie bereits weiter oben dargelegt, sind die Bedingungen, unter welchen wir Wissen zuzuschreiben bereit sind, konstitutiv für unseren Begriff des Wissens. Der westliche Wissens-Begriff unterscheidet sich also, wenn wir den Studien der Vertreterinnen und Vertreter der Experimentellen Philosophie vertrauen können, vom ostasiatischen Wissens-Begriff. Wer fragt, wieso wir unseren Wissens-Begriff bei der Beantwortung der Frage, was Wissen ist, gegenüber dem ostasiatischen »bevorzugen« sollten, verkennt, dass die

73 Nichols/Knobe 2008, S. 11.

74 Nadelhoffer/Nahmias 2007, S. 128. 
Frage »Was ist Wissen? « bereits entweder in unserem oder im ostasiatischen Begriffssystem gestellt wird. Wenn wir uns für das Wesen des Phänomens interessieren, auf das wir uns mit »Wissen « beziehen, sollten wir der Untersuchung unseren Begriff zugrunde legen; wenn wir uns für das Wesen des Phänomens interessieren, auf das Ostasiatinnen und Ostasiaten mit »Wissen « (oder der Entsprechung davon in einer ostasiatischen Sprache) Bezug nehmen, dann sollten wir der Untersuchung ihren Begriff zugrunde legen. Es ist nicht so, dass wir eine Theorie darüber haben, was Wissen ist, und die Ostasiatinnen und Ostasiaten eine Theorie darüber haben, was Wissen ist, und eine der beiden Theorien ist adäquater als die andere. Sondern »Was ist Wissen?« hat je nachdem, welcher Wissensbegriff zugrunde gelegt wird, verschiedene Bedeutungen, und die Analyse unseres Wissensbegriffs beantwortet eine andere Frage als die Analyse des ostasiatischen Wissensbegriffs. ${ }^{75}$

Wäre es nun aber nicht doch denkbar, dass einer der beiden Wissensbegriffe, der westliche oder der ostasiatische, besser ist als der andere? Erfasst vielleicht einer der beiden ein wichtigeres Phänomen oder ist besser dazu geeignet, auf das Bezug zu nehmen, was Wissen wirklich - sprach-unabhängig ist? Eine ausführliche Antwort auf diese Frage hängt damit zusammen, was von wittgensteinianisch geprägten Philosophinnen und Philosophen unter den Stichworten der Autonomie der Grammatik und des begrifflichen Relativismus diskutiert wird, und würde den Rahmen der vorliegenden Abhandlung sprengen. Hier nur ein paar Bemerkungen: Erstens, Wissen ist keine natürliche Art, es ergibt also keinen Sinn, zu behaupten, dass wir das Phänomen des Wissens mit unserer Sprache entweder treffen oder daran vorbeizielen. Zweitens, wenn wir ein Gedankenexperiment unterbreitet bekommen und beurteilen sollen, ob es ein Fall von Wissen ist oder nicht, dann beurteilen wir nicht, ob etwas Zusätzliches, das in der Fallbeschreibung nicht erwähnt worden ist, auch noch vorliegt, d. h. auch noch »da ist« oder nicht, sondern wir entscheiden uns für eine bestimmte Art und Weise, das, was durch die Fallbeschreibung gegeben ist, zu beschreiben. Es gibt keine Tatsache des

75 Tatsächlich sollte also in philosophischen Abhandlungen im Falle von divergierenden Begriffen jeweils einer der verschiedenen Begriffe privilegiert behandelt werden, nämlich derjenige, der dem zur Diskussion stehenden Problem zugrunde liegt. Dies gilt nicht nur bei kulturellen Unterschieden, sondern auch etwa bei Unterschieden zwischen einem Alltags- und einem Fachbegriff. Um ein Beispiel aus der Einleitung des vorliegenden Buchs aufzugreifen: Wenn ich mich dafür interessiere, was es bedeutet, jemanden über etwas zu informieren, wird es mir nicht helfen, die Gebrauchsweise des Ausdrucks »Information«, die in den Informationswissenschaften etabliert ist, zu untersuchen. Umgekehrt dürfen wir uns, wenn wir beschreiben, was »Information« in den Informationswissenschaften bedeutet, aber auch nicht von unserem Alltagsbegriff beirren lassen. 
Vorliegens oder Nicht-Vorliegens von Wissen unabhängig von unserem Begriff des Wissens. Drittens, innerhalb von bestimmten Grenzen ergibt es tatsächlich Sinn, von der Nützlichkeit eines Begriffs zu sprechen. Beispielsweise wurde der deutsche Begriff der Schadenfreude ins Englische übernommen, weil es bequemer ist, "Schadenfreude« zu sagen als immer auf die Umschreibung »taking pleasure in other people's suffering « zurückzugreifen. Tatsächlich drückt aber »taking pleasure in other people's suffering « denselben Begriff aus wie »Schadenfreude«, weshalb es eigentlich nicht ganz zutreffend ist, dass bei der Einführung des Wortes »Schadenfreude« ein neuer Begriff ins Englische eingeführt wurde.

Meines Erachtens lässt sich nicht stichhaltig dafür argumentieren, dass unser Wissensbegriff in irgendeiner Weise nützlicher ist als der ostasiatische Wissensbegriff (oder umgekehrt). Und tatsächlich lässt sich der ostasiatische Wissensbegriff ja im Deutschen ausdrücken: »ein Fall von Wissen oder von einer wahren, gerechtfertigten Überzeugung, die auf einer falschen Überzeugung beruht«. Umgekehrt könnten die Ostasiatinnen und Ostasiaten unseren Begriff mit einer Formulierung der Art »Wissen, das nicht auf einer falschen Überzeugung beruht« ausdrücken. Im entscheidenden Sinn besitzen wir also auch den ostasiatischen Wissensbegriff, und die ostasiatischen Sprecherinnen und Sprecher besitzen auch unseren.

\section{5} Schluss

Im vorliegenden Kapitel habe ich unter anderem dafür argumentiert,

- dass sich Vertreterinnen und Vertreter der Ordinary Language-Philosophie nicht auf Intuitionen berufen, sondern auf ihre Sprachkompetenz,

- dass in der Experimentellen Philosophie des Öfteren die Tendenz anzutreffen ist, begriffliche Fragen mit empirischen Fragen zu verwechseln (und zwar nicht nur in dem Sinn, dass angenommen wird, man könne (oder müsse) begriffliche Fragen mittels empirischer Methoden beantworten),

- dass Aussagen der Form »Wir würden sagen, dass ...« nicht hypothetisch und eher normativ als deskriptiv aufzufassen sind,

- dass häufig nicht festgelegt ist, wie philosophisch interessante Begriffe auf exotische Fälle anzuwenden sind, und

- dass die von den Vertreterinnen und Vertretern der Experimentellen Philosophie entdeckte Diversität in den Reaktionen auf Gedankenexperimente teilweise darauf zurückzuführen ist, dass verschiedene Probandinnen und Probanden unterschiedliche Begriffe (des Wissens, der Absichtlichkeit usw.) besitzen. 
Vor allem habe ich aber zu zeigen versucht,

- dass wir ohne die Befragung anderer Sprecherinnen und Sprecher beurteilen können, welche Regeln zur Verwendung sprachlicher Ausdrücke wir befolgen, und

- dass der Fokus auf unsere eigenen Begriffe in der Philosophie keiner ungerechtfertigten Privilegierung entspricht.

Die besprochenen Argumente, die vonseiten der Experimentellen Philosophie gegen die Ordinary Language-Philosophie vorgebracht worden sind, haben sich alle als nicht stichhaltig erwiesen. 